\title{
Le temps long du dialogue entre médecins et philosophes
}

Claire Crignon ${ }^{1}$, David Lefebvre ${ }^{2}$

Sans prétendre proposer un parcours exhaustif du dialogue entre médecins et philosophes, depuis l'Antiquité jusqu'à la période contemporaine, l'ouvrage collectif Médecins et philosophes : une histoire [1] propose une incursion portant sur les échanges entre ce qui nous apparaît aujourd'hui comme deux disciplines bien distinctes. Il propose un panorama large de ce dialogue, de Platon à Daniel Lagache (1903-1972) ou Favez-Boutonier (1903-1994), sans viser l'exhaustivité, puisque des pans importants de la tradition médico-philosophique se trouvent traités en passant - les philosophes-médecins présocratiques, Hippocrate, la médecine alexandrine d'Hérophile et d'Érasistrate, la tradition médiévale latine, etc. II était impossible dans les limites d'un seul ouvrage de consacrer une étude à chaque auteur. L'objectif était ici de se concentrer sur le versant épistémologique du dialogue entre médecine et philosophie sur le temps le plus long possible, ce qui condamnait d'emblée à des choix ; nous voudrions ici revenir sur les raisons de ce choix.

La première question qui peut survenir à la lecture du titre et de l'avantpropos est la suivante : pourquoi retracer ce dialogue sur un temps aussi long, alors même que ce que nous nommons «médecine » et «philosophie » n'a certainement pas le même sens pendant l'Antiquité, au Moyen-âge, à la Renaissance, durant
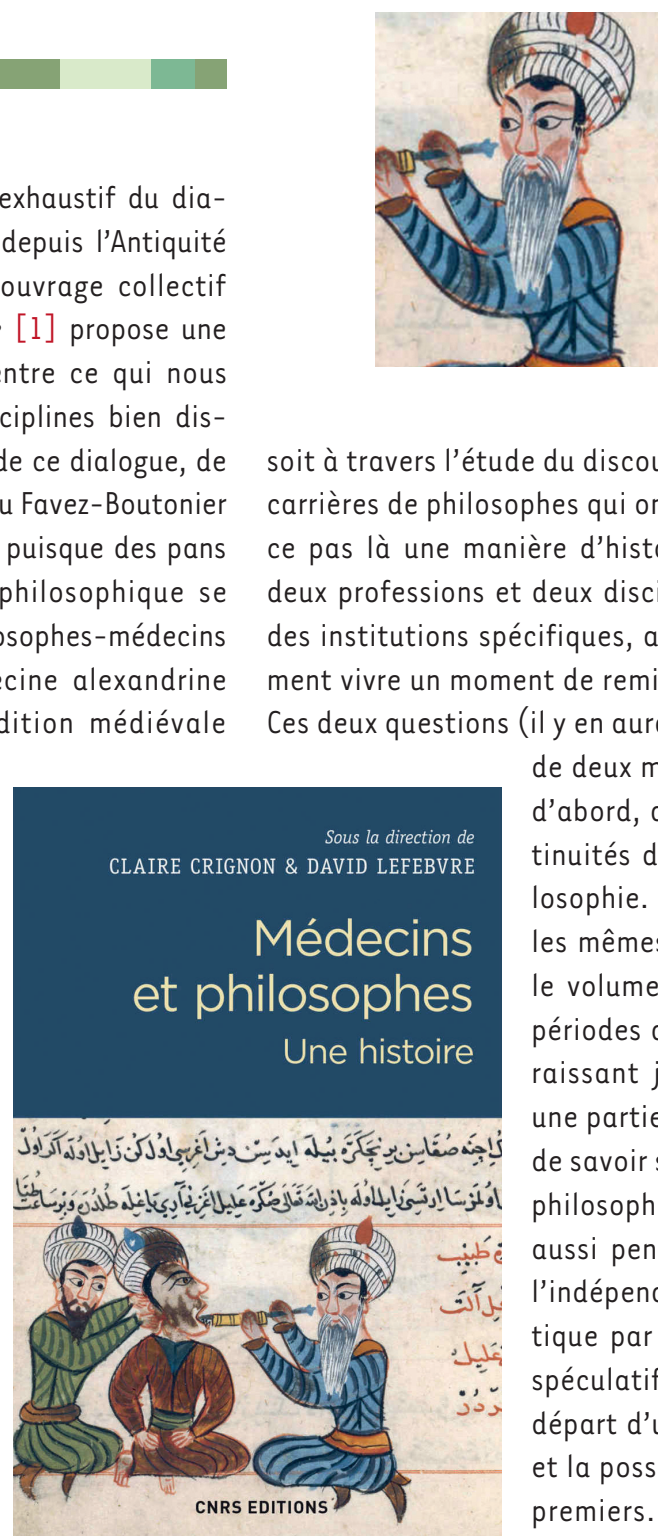

${ }^{1}$ Sorbonne Université et Sciences Normes Démocratie,

1 rue Victor-Cousin, 75005 Paris, France.

${ }^{2}$ Sorbonne Université et Centre Léon Robin, UMR 8061 du CNRS, Paris,

France.

clairecrignon@gmail.com

david.lefebvre@sorbonne-universite.fr

soit à travers l'étude du discours sur le «philosophe-médecin » ou des carrières de philosophes qui ont choisi de «se faire médecins ». N'estce pas là une manière d'historiciser l'idée d'un partage strict entre deux professions et deux disciplines correspondant à des études et à des institutions spécifiques, alors même que nous semblons actuellement vivre un moment de remise en question de ces séparations?

Ces deux questions (il y en aurait d'autres) seront pour nous l'occasion de deux mises au point. II nous semble important, d'abord, de revenir sur les continuités ou discontinuités dans le dialogue entre médecine et philosophie. Les enjeux du dialogue ne peuvent être les mêmes tout au long de la période que couvre le volume. II y a certes une continuité entre les périodes antique et moderne : la médecine apparaissant jusqu'à cette dernière période comme une partie de la philosophie naturelle, la question de savoir si elle doit chercher ses principes dans la philosophie est certes restée cruciale. Mais c'est aussi pendant la période moderne que s'affirme l'indépendance de la médecine comme savoir pratique par rapport à la philosophie comme savoir spéculatif, indépendance qui constitue le point de départ d'un discours critique sur la métaphysique et la possibilité de remonter jusqu'à des principes premiers. Enfin, il y a une différence de taille entre les périodes moderne et contemporaine. les périodes moderne et contemporaine? Une seconde question peut se poser relativement au parti-pris opéré de personnaliser ce dialogue, en plaçant la focale sur les personnages du médecin et du philosophe, que ce

Vignette (Photo @ CNRS Éditions).
Si, aujourd'hui, la médecine ou l'éthique médicale sont considérées comme des objets à part entière, qui nécessitent une enquête philosophique spécifique et une spécialisation que les philosophes peuvent ou non entreprendre, les philosophes de la période moderne ne font pas cette séparation. Ils considèrent la médecine comme partie intégrante du projet philosophique. Loin de constituer l'objet d'un savoir extérieur 
à l'enquête philosophique, la médecine est pour eux indispensable à la poursuite de la santé et de la longévité, et donc aussi nécessaire pour accéder à une vie bonne et heureuse, tant sur le plan éthique que politique. La médecine constitue le point de départ d'un régime qui s'applique aussi bien au corps qu'à l'esprit et qui s'inscrit dans le projet plus large d'une connaissance de l'homme tout entier. L'éthique, loin d'être appelée en renfort pour prendre la mesure des enjeux philosophiques des pratiques ou décisions médicales, est ici consubstantielle à la définition de la philosophie comme soin ou souci de soi [2]. La «philosophie médicale » des modernes (au XvII siècle) n'est donc pas la «philosophie de la médecine » de la période contemporaine.

Il ne sera pas superflu, par ailleurs, de s'arrêter sur les évolutions récentes qu'ont connues non seulement les études de médecine mais aussi la recherche médicale, et sur les demandes adressées aux sciences humaines et sociales de participer à ces évolutions. Aujourd'hui, on ne parle plus de favoriser le dialogue entre «médecins » et «philosophes », mais on en appelle à une fusion des deux professions ou disciplines à l'intérieur d'un ensemble plus vaste qui englobe la philosophie, les lettres, les sciences sociales, l'art et que I'on nomme les «humanités médicales » ${ }^{1}[3-5]$. Toute la question est alors de savoir ce que l'on entend ici exactement par « humanités » et si cette expression d'humanités médicales suppose l'institution d'un rapport de concurrence entre lettres, sciences humaines et sociales d'un côté, médecine et sciences de l'autre, ou au contraire d'intégration d'une démarche à une autre. Or cette intégration, loin de s'imposer comme une évidence, exige la mise en œuvre d'un réel effort d'interrogation critique sur les pratiques d'enseignement et de recherche. Cet effort de réflexion critique nécessite du temps et du recul, temps et recul que les exigences liées à l'urgence des modalités de financement de la recherche en médecine, et désormais en sciences humaines et sociales, ne rendent pas toujours possibles. Ces difficultés peuvent être parfois à l'origine de ce qui apparaît bien souvent comme un « dialogue de sourd » entre médecins et philosophes, mais elles représentent aussi un nouveau défi à surmonter pour les années à venir.

\section{Continuités et discontinuités du dialogue entre médecins et philosophes}

Philosophie et médecine n'ont cessé d'entretenir des relations extrêmement étroites, mais il serait trompeur de faire comme si la nature de cette proximité était restée la même depuis l'Antiquité. La continuité de la relation est en partie fictive et c'est en marquant les différences que l'on peut saisir l'histoire de cette relation et la nouveauté de ce qui se joue actuellement. S'il existe une «demande » de la part de certains acteurs de la médecine en direction de la philosophie, et plus généralement des «humanités», il reste que le savoir médical lui-même ne se considère plus comme scientifiquement dépendant de la philosophie, même si la philosophie peut contribuer

${ }^{1}$ Cette appellation a d'abord été diffusée dans le monde anglo-saxon. Pour un panorama sur son apparition et son succès, voir [3-5]. au renouvellement de certaines théories ou approches thérapeutiques ${ }^{2}$. Cela dit, d'une part, la médecine peut vouloir s'appuyer, dans certaines de ses pratiques, sur des savoirs extérieurs à la médecine stricto sensu, et, d'autre part, la question se pose encore de définir la nature de la relation entre la médecine et la biologie ou la chimie; or l'une et l'autre étaient jadis des parties intégrantes de la philosophie. La philosophie, de son côté, a cessé de considérer la médecine comme le savoir privilégié à examiner dans le cadre d'une recherche sur la nature de la science et de sa méthode. Mais la philosophie continue de trouver matière à penser dans les pratiques médicales et le discours des médecins. C'est sur ces deux points que philosophie et médecine ont forgé une histoire commune dès l'Antiquité : (1) la question qui a traversé la réflexion des médecins depuis Hippocrate était celle de savoir si la médecine devait ou non prendre ses principes dans la philosophie, en particulier parce que cette dernière contenait une philosophie de la nature; Hippocrate, du moins dans le traité De l'ancienne médecine, a choisi la séparation ${ }^{3}$ [6]. Mais pour Aristote et Galien, même si la médecine et la philosophie sont deux disciplines différentes, la physique (qui relève de la philosophie ou qui constitue l'objet commun de la médecine et de la philosophie) est au fondement de la connaissance du corps, sain ou malade, et de son traitement. (2) Depuis Platon, la médecine est un paradigme qui intervient dans toutes les parties de la philosophie, notamment la théorie de la science et l'éthique.

Ces deux types de rapport entre philosophie et médecine sont de nature très différente, et ils sont, en partie seulement, indépendants l'un de l'autre. Dans le premier cas, le problème est celui des rapports structurels et hiérarchiques entre la médecine et la philosophie. II concerne les philosophes comme les médecins ; c'est l'annexion de la médecine à la philosophie, l'indépendance de la médecine et son statut qui sont en cause ; dans sa forme classique en tout cas, cette question suppose l'existence d'une science de la nature qui dépend de la philosophie. La subordination de la médecine à la philosophie, ou la coordination des deux, encourage une conception technique de la médecine (la médecine est un art, voire une simple pratique empirique, qui trouve ses principes dans la science philosophique), tandis que la séparation va dans le sens de l'affirmation de la scientificité propre de la médecine ${ }^{4}$. Dans le second

\footnotetext{
Nous reviendrons sur ce point dans la seconde partie de l'article.

3 Sur la diversité de ce qu'on appelle la « collection hippocratique », on pourra se reporter dans le volume à l'article de P. Pellegrin [6].

${ }^{4}$ On ne peut qu'évoquer ici la position des médecins empiriques selon laquelle la médecine n'est ni une science ni même un art.
} 
type de rapport mentionné, la relation entre médecine et philosophie est en grande partie unilatérale : c'est la philosophie qui utilise - à titre de modèle (ou de paradigme) ou d'illustration - la médecine, comme elle peut le faire également à d'autres fins avec l'architecture ou l'art de la navigation, ou encore, de manière générale, c'est la philosophie qui va puiser dans la médecine des objets philosophiques. Ces deux types de rapports (que l'on peut qualifier d'architectonique, pour le premier, et de paradigmatique, pour le second) ne sont pas sans relation; ils rencontrent, par des voies différentes, les mêmes difficultés sur la nature de la médecine, dont la plus récurrente : est-elle un art ou une science? Mais si la philosophie trouve matière à penser dans la médecine, c'est aussi parce que cette dernière développe une réflexion propre sur elle-même, sa pratique, ses moyens, etc. Cette pensée méta-médicale de la médecine, particulièrement riche dans l'Antiquité, s'enracine dans les discussions philosophiques de son temps et cherche en partie à concurrencer ces dernières afin de doter la médecine d'une méthode propre. Les discussions, vives pendant la période hellénistique de la philosophie (à partir du $\|^{e}{ }^{e}$ siècle av. J.-C.), entre les différentes « sectes médicales », « dogmatique », « empirique et méthodiste » en sont un exemple ${ }^{5}$.

Sur la première dimension évoquée (la question architectonique), il faut préciser les termes dans lesquels la question du «médecin philosophe » s'est posée. Du côté des philosophes, Aristote est le premier à examiner le problème des rapports entre médecine et philosophie ; il le fait d'une manière ambiguë mais qui restera cependant fondamentale pour toute la tradition philosophique. On tire de plusieurs textes du corpus aristotélicien issus de ses traités psychophysiologiques l'idée que la médecine trouve ses principes dans la partie de la philosophie naturelle qui s'occupe des objets les plus particuliers (les propriétés communes au corps et à l'âme des vivants et notamment des êtres humains). D'où la célèbre formule selon laquelle « là où s'arrête la philosophie, commence la médecine ». Mais, étant donné nos doutes sur la nature descriptive ou normative des textes aristotéliciens concernés, il est difficile de savoir si Aristote imaginait une articulation précise entre ces deux disciplines : constatait-il seulement une relation entre elles chez certains auteurs, mais sans la prendre à son compte, ou bien au contraire l'appelait-il de ses vœux ? [7, 8]. La tradition aristotélicienne, grecque, latine, arabe, jusqu'aux médecins de la Renaissance tentera de clarifier cette position de manière plus ou moins systématique, ce qui reviendra à préciser le statut de la médecine comme science pratique, discipline à mi-chemin entre la science et l'art. Certains, comme Avicenne, s'efforceront de concilier la dépendance de la médecine vis-à-vis de la science philosophique, et le statut scientifique de la médecine. D'autres, comme Al-Fārābī ( $\mid X^{e}-x^{e}$ siècles) ou Averroès ( $x \|^{e}$ siècle), situeront la médecine dans une relation de subordination théorique à la philosophie naturelle, en

\footnotetext{
${ }^{5}$ Les médecins dogmatiques posent des dogmes ou des opinions. Considérant qu'il est possible d'accéder aux causes des maladies par le biais d'inférences logiques, ils assignent au savoir médical une ambition rationnelle forte. Les médecins empiriques considèrent quant à eux qu'il n'est pas possible de connaître les causes des maladies et ils se fondent sur l'observation des symptômes. Les méthodiques tout en rejetant la possibilité d'un accès aux causes cachées ne voient pas non plus dans l'expérience la clé du savoir. Ils considèrent que toutes les maladies viennent soit d'un état de resserrement, de relâchement ou d'un mélange entre ces deux états.
}

soulignant que la médecine doit rester un art, dépourvu de certitude absolue, toujours au contact de réalités particulières, et dont la fin est seulement de soigner. Galien, quant à lui, s'oppose sans doute à la position séparatiste illustrée par le traité hippocratique $D e$ l'ancienne médecine qui veut émanciper l'art médical de «postulats » physiques, c'est-à-dire d'une étiologie simplificatrice représentée par les couples de contraires traditionnels (chaud/froid, sec/humide). Mais il ne pense pas que la médecine doive se fonder sur un savoir étranger qu'elle accepterait de manière passive, la physique des éléments et des humeurs. Pour Galien, la médecine est une science. Médecine et philosophie collaborent dans la constitution d'une science de la nature; sur un certain nombre de questions physiques, les meilleurs médecins et les meilleurs philosophes recherchent la même chose et peuvent déboucher sur les mêmes conclusions (par exemple, que l'homme n'est pas constitué d'une seule et même nature). L'« excellent médecin » fera donc de la philosophie naturelle, mais ce n'est rien d'autre en fait que de la médecine. Jusqu'à un certain point, les deux font la même chose sous des noms différents. Jusqu'à un certain point seulement, en effet, puisque Galien a une claire conscience des limites entre les deux disciplines - l'« excellent médecin » même philosophe ne discutera pas de l'essence de l'âme, de la création du monde ou de l'existence du vide extra-mondain car il n'en a pas besoin pour soigner $[9,10]$.

Passons maintenant au second niveau dont nous parlions. La philosophie utilise la médecine comme une matière philosophique. Plusieurs auteurs y reviennent dans «Médecins et philosophes. Une histoire ». L'historien de la philosophie antique Riccardo Chiaradonna (Université Roma Tre, Rome, Italie) examine de manière détaillée de quelle façon Galien s'est intéressé de près aux rapports entre les sensations (du médecin ou du patient), leur verbalisation et leur conceptualisation, à une époque où la médecine, dépourvue de moyens d'observation interne, était tributaire des comptes rendus des patients [11]. Galien montre ainsi sa conscience de l'importance des limites du langage dans la pratique et le discours des médecins. II s'engage ainsi sur des questions caractéristiques de la philosophie hellénistique d'une manière qui intéresse le philosophe et l'histoire de la philosophie, et il fait bien lui-même œuvre de philosophe en un sens, même s'il n'examine pas ces questions pour elles-mêmes mais toujours dans l'optique du traitement et du soin. De manière plus générale, l'un des points fondamentaux qui a retenu le plus l'attention des philosophes est l'articulation entre le niveau théorique ou scientifique (celui de la connaissance des 
principes les plus généraux, dérivés, selon certains, de la connaissance philosophique) et celui de la pratique ou, comme on le dit parfois, de l'« application ». Comment penser cette relation dans laquelle on situe aussi parfois l'origine de l'échec du médecin ? Le cas de Platon est ici exemplaire : ce dernier construit dans ses dialogues une distinction entre trois types de médecine (selon son degré de rationalité et son rapport au cas particulier). II cherche ainsi en fait à donner un paradigme ou un modèle de la situation même du philosophe : ce dernier, en effet, quelle que soit sa connaissance théorique des Formes, ne peut être assuré de réussir dans ses entreprises pratiques, notamment législatives [12]. La médecine est un modèle qui permet à la philosophie de représenter sa propre manière d'articuler la saisie de l'intelligible et de l'éternel et son action dans la réalité sensible et contingente.

\section{La médecine en quête d'humanité(s) : un dialogue de sourds entre médecins et philosophes?}

Qu'en est-il aujourd'hui des deux types de rapports envisagés précédemment pour penser le rapport entre philosophie et médecine, architectonique et paradigmatique ? L'idée que la médecine puisse aller chercher dans la philosophie des fondements pour comprendre la nature du corps humain sain ou malade ne paraît plus pertinente. Pourtant, certains chercheurs considèrent que la recherche en philosophie de la biologie et de la médecine peut et doit avoir une incidence sur la recherche médicale [13]. Quatre types d'apports peuvent être répertoriés: la philosophie contribue à la recherche médicale par 1) un travail de clarification des concepts scientifiques ; 2) un examen critique des énoncés et des méthodes scientifiques ; 3 ) la formulation de nouveaux concepts et de nouvelles théories; et 4) par la promotion du dialogue entre les différentes sciences (médecine, biologie, physique, chimie, mais aussi les sciences humaines et sociales) et entre la science et la société. On peut voir dans la première tâche un objectif qui s'inscrit dans la lignée des déclarations du philosophe et historien de la médecine Georges Canguilhem, dans l'introduction de son Essai sur quelques problèmes concernant le normal et le pathologique, lorsqu'il insistait sur l'importance du travail de clarification à effectuer concernant l'usage des concepts de normal et de pathologique en médecine. La troisième tâche renvoie au champ de l'épistémologie des sciences médicales et à l'essor actuel de la philosophie de la médecine. La seconde est certainement plus originale et ambitieuse, puisqu'elle suggère que le chercheur en philosophie de la médecine ou de la biologie est susceptible d'introduire de nouveaux concepts ou théories au sein même de la recherche médicale, comme l'ont fait deux philosophes contemporains de la biologie et de la médecine, Thomas Pradeu en redéfinissant le concept d'identité à partir de recherches en immunologie, ou Lucie Laplane [13] en proposant une approche interdisciplinaire du concept de cellule souche et en démontrant l'incidence des différentes caractérisations de ce concept sur le choix d'une stratégie thérapeutique contre le cancer. Toutefois et même si l'on peut considérer que, dans certains domaines, la recherche en philosophie de la biologie et de la médecine contribue à proposer de nouvelles théories et de nouveaux concepts, on ne se tourne plus vers la philosophie en général comme vers une discipline susceptible de venir fonder la recherche médicale en tant que telle. La philosophie intervient ici non plus au titre d'une science des fondements ou des principes, fournissant les racines permettant à l'arbre de la connaissance (physique, médecine, morale et technique) de se déployer, mais comme un domaine de recherche fortement spécialisé qui a acquis une indépendance par rapport à la science médicale et qui est susceptible de venir remettre en question certains concepts tout en proposant des théories nouvelles. D'une certaine manière, ce n'est plus vers la philosophie que le médecin se tourne pour chercher un fondement à la compréhension des corps naturels, mais c'est plutôt la matière médicale elle-même qui, en faisant l'objet de recherches expérimentales menées par le philosophe, devient un terreau permettant à ce dernier de définir de nouveaux concepts et de contribuer aux avancées de la médecine.

Mais revenons un instant sur le quatrième type d'apport : la promotion d'un dialogue entre les sciences dites « dures » et les sciences humaines et sociales, l'interaction entre science et société. Ce point nous permettra de revenir sur le second type de rapport envisagé entre médecine et philosophie, le rapport dit paradigmatique. Ici encore, on peut avoir le sentiment que la relation s'est inversée. Loin de chercher dans la médecine un modèle ou une illustration pour la philosophie, la médecine semble se tourner vers la philosophie comme vers un modèle de sagesse, comme la discipline par excellence susceptible de répondre à un besoin de plus en plus grand d' « humaniser » le soin et les pratiques thérapeutiques. La demande concerne plus spécifiquement l'éthique médicale ou la bioéthique que la philosophie en tant que telle. Et elle peut aussi s'adresser à la psychologie, à la sociologie, à l'anthropologie ou aux sciences de l'information et de la communication. Néanmoins, la philosophie se trouve sollicitée au premier chef, d'une part en raison de l'essor considérable qu'a connu le domaine de la bioéthique depuis l'après-Seconde Guerre mondiale, d'autre part du fait de la médiatisation des «affaires » et de l'accélération importante des progrès accomplis dans le domaine du savoir et des techniques médicales. Jusqu'à une période relativement récente (I'après-Seconde Guerre mondiale), la philosophie constituait une sorte de couronnement des études de médecine, comme le montre le cas de ces «médecins-philosophes » ayant suivi un double parcours universitaire en philosophie (agrégation, École Normale Supérieure de la rue d’Ulm) et en médecine [14]. 
Les évolutions récentes de la recherche en médecine (le fait en particulier que tous les appels à projets doivent désormais inclure un volet relatif aux sciences humaines et sociales) tout comme la réforme en cours des études de médecine (fin du numerus clausus, refonte de la $P A C E S^{6}$, possibilité nouvelle d'accéder à des études de médecine à partir d'autres formations disciplinaires, via des passerelles et des mineures santé, enfin présence obligatoire d'un représentant des sciences humaines et sociales dans le jury de la PACES) semblent bien traduire cette demande d'un dialogue plus étroit entre les sciences d'un côté et, de l'autre, les sciences humaines et sociales, désignées par l'étiquette commode d'《humanités ». Cette demande est certainement le résultat de la spécialisation et de la séparation entre les différents domaines du savoir qui commence à devenir visible dès la fin de la période moderne et qui culmine au XVIII siècle avec l'idée kantienne d'un conflit entre les facultés [15]. Chez Locke déjà, à la fin du XvII siècle, on trouve cette affirmation maintes fois répétée que le philosophe ne doit pas «se mêler de médecine », mais seulement souhaiter à la pratique médicale de réussir [16]. Le philosophe n'est pas là pour guider le médecin ni pour s'immiscer dans sa pratique. Les rôles sont donc clairement définis et départagés à partir de la période moderne et la spécialisation ne fera qu'accentuer ce processus.

Pourtant, comme le montrent les articles du volume consacrés à la période moderne et contemporaine, le besoin d'une « alliance » entre médecins et philosophes n'a cessé de s'exprimer depuis «l'alliance épistémologique » dessinée par P. J. G. Cabanis [17] jusqu'aux travaux de F. Dagognet, de G. Canguilhem et de K. Jaspers [18, 19]. L'épilogue de l'ouvrage, rédigé par A.M. Moulin, médecin et philosophe, permet de prendre la mesure des difficultés de ce type de parcours, tout en montrant aussi le bénéfice d'un « exercice philosophique » de la médecine capable de sortir de son domaine de spécialité, ouvert au dialogue, conscient de la marge d'incertitude et d'erreur inhérente à l'art médical [20]. Comment alors penser et pratiquer ce dialogue entre médecine et sciences humaines et sociales sans revenir à cette position surplombante assignée à la philosophie, sans non plus lui assigner la tâche de venir « humaniser » une relation entre médecins et patients dont la qualité serait compromise par l'hyperspécialisation et la technicisation des pratiques? Depuis Galien jusqu'au médecin portugais Rodrigo de Castro dans son Medicus Politicus (Hambourg, 1614) [21], les médecins n'ont eu de cesse de souligner l'importance d'une formation médicale qui intègre non seulement les connaissances scientifiques les plus pointues (physiologie, biologie, chimie), mais aussi les lettres classiques et ce que l'on appelait alors « les humanités » (la philosophie, l'histoire, les langues, l'art). Cette insistance ne visait pas à proposer de faire de ces disciplines un modèle pour la pratique médicale; elle cherchait à marquer la nécessité d'un recul critique sur les pratiques, le besoin d'une capacité à les interroger sur le temps long et non pas simplement dans l'urgence des situations pratiques dramatiques ou au rythme de l'éclairage médiatique des scandales et des affaires qui ponctuent l'actualité. Cette réflexion et ce recul critique intervenaient de l'intérieur même de la médecine; elle était à l'œuvre dans les écrits et la

\footnotetext{
${ }^{6}$ Première année commune aux études de santé.
}

pensée des médecins. Mais ce dialogue critique pouvait aussi conduire à remettre en question les définitions en usage ou les critères traditionnels de distinction entre humanité et animalité [22]. Loin de fournir des solutions toutes faites ou consensuelles, il implique donc une prise de risque, mais aussi la capacité à reconnaître les limites de ce que les humanités peuvent apporter à la médecine : certainement pas I'humanisation qui lui manquerait et qu'elle pourrait fournir comme un « supplément d'âme » ou comme une réponse à la crise du système de santé. «Le premier geste de l'approche humaniste - c'est-à-dire philosophique et historique consiste à mettre en lumière la démarche scientifique » propre à la médecine, écrivait Jean Starobinski dans son «Plaidoyer pour des humanités médicales » au début de ce siècle [23]. La tension, repérée quinze ans auparavant par l'historien de la médecine Mirko Gremk, entre la recherche scientifique et son application technique, d'une part, les valeurs humanistes et les contraintes sociales, d'autres part, était alors toujours d'actualité, comme elle l'est encore aujourd'hui. On a sans doute encore plus de motifs aujourd'hui de considérer que seule une approche interdisciplinaire peut nous permettre de démêler l'« épineux problème » dont parlait Grmek [24]. Á quoi nous proposons d'ajouter le temps long de la réflexion sur les rapports entre « les humanités » et la médecine.

The long time of dialogue between doctors and philosophers $\diamond$

\section{LIENS D'INTÉRÊT}

Les auteurs déclarent n'avoir aucun lien d'intérêt concernant les données publiées dans cet article.

\section{RÉFÉRENCES}

1. Crignon C, Lefebvre D. Médecins et philosophes : une histoire. Paris : CNRS Éditions, 2019 : p. 509.

2. Smith J. Early modern medical eudaimonism. In : Early modern medicine and natural philosophy. Distezweig P, Goldberg B, Ragland ER (eds). Springer, $2016: 325-41$.

3. Gaille M. Tableau des humanités médicales. Recension de Fantini B, Lambrichs LL (dir.) In : Histoire de la pensée médicale contemporaine évolutions, découvertes, controverses. Paris : Seuil, 2014. https://laviedesidees.fr/Tableau-des-humanites-medicales.htlm

4. Lambrichs LL. La médecine occidentale en quête d'humanité(s). In : Histoire de la pensée médicale contemporaine. Paris : Seuil. $2014 ; 26$ : 455-72.

5. The Edinburgh companion to the critical medical humanities. Whitehead A, Woods A (eds). Edinburgh University Press, 2016.

6 Pellegrin P. Les écoles empiriques et méthodiste : une médecine positive? In : Médecins et philosophes : une histoire. Paris : CNRS Éditions, 2019 : 85-108.

7 Lefebvre D. Physique et médecine chez Aristote : subordination, séparation, communauté. In : Médecins et philosophes : une histoire. Paris : CNRS Éditions, 2019 : 51-84.

8 Lo Presti R. Entre aristotélisme médical et médecine aristotélisante : le rapport entre médecine et philosophie dans les commentaires italiens du Xvi ${ }^{e}$ siècle au De sensu d'Aristote. In : Médecins et philosophes : une histoire. Paris : CNRS Éditions, $2019: 197-224$. 


\section{RÉFÉRENCES}

9 Boudon-Millot V. Galien de Pergame ou le médecin qui voulait se faire Philosophe. In : Médecins et philosophes : une histoire. Paris : CNRS Éditions, 2019 : 109-28.

10 Koetschet P. Abū Bakr al-Rāzi contre Galien, ou comment s'élever du niveau des médecins à celui des philosophes. In : Médecins et philosophes : une histoire. Paris : CNRS Éditions, 2019 : 147-70.

11 Chiaradonna R. Langage ordinaire et connaissance médicale selon Galien. In : Médecins et philosophes : une histoire. Paris: CNRS Éditions, 2019 : 129-46.

12 Auffret T. Approximation, métrétique et stochastique : le modèle platonicien de la médecine. In : Médecins et philosophes : une histoire. Paris : CNRS Éditions, 2019 : 19-50.

13 Laplane L. Mantovani P. Adolphs R, et al. Opinion: why science needs philosophy. Proc Natl Acad Sci USA 2019 : 3948952

14 Dupouy D, Trochu T. Pourquoi des philosophes de la République se sont-ils faits médecins? (France 1888-1958). In : Médecins et philosophes : une histoire. Paris : CNRS Éditions, 2019: 321-64.

15 Buchenau S. Kant, la médecine et le conflit des facultés. In : Médecins et philosophes : une histoire. Paris : CNRS Éditions, $2019: 269-93$

16 Crignon C. Disputes médicales et controverses sectaires. Les enjeux pratiques de la réflexion de Locke médecin sur la diversité. In : Médecins et philosophes : une histoire. Paris : CNRS Éditions, 2019 : 245-68.

17 Gaille M. D’indispensables services rendus : l'alliance épistémologique. In : Médecins et philosophes : une histoire. Paris : CNRS Éditions, 2019 : 295-319.
18 Durrive B. La technique : point de rencontre entre philosophie et médecine chez Georges Canguilhem. In : Médecins et philosophes : une histoire. Paris : CNRS Éditions, 2019 : 365-88.

19 Basso $\varepsilon$. Karl Jaspers et la nouvelle philosophie de la psychiatrie. In Médecins et philosophes : une histoire. Paris : CNRS Éditions, 2019 : 389-409.

20 Moulin AM. Philosophie et médecine : l'impossible choix. In : Médecins et philosophes : une histoire. Paris : CNRS Éditions, 2019 :411-30.

21 Cardoso A. Le médecin selon Rodrigo de Castro où la disciplina mentis du médecin parfait. In : Médecins et philosophes : une histoire. Paris : CNRS Éditions, 2019 : 225-44.

22 Andrault R, Buchenau S, Crignon C. et al. Médecine et philosophie de la nature humaine de l'âge classique aux Lumières. Paris : Garnier, 2014.

23 Starobinski J. Plaidoyer pour des humanités médicales. In : Littérature et médecine ou les pouvoirs du récit. Actes du colloque organisé par la BPI les 24 et 25 mars 2000. Préface. Danou G (ed). Paris : Centre Pompidou, 2000.

$24 \mathrm{Grmek}$ M. Médecine et épistémologie : transformation du savoir sur la santé et la maladie. Discours d'ouverture de la conférence de Pérouse. Hist Phil Life Sci 1988. $10: 3-6$.

TIRÉS À PART

C. Crignon

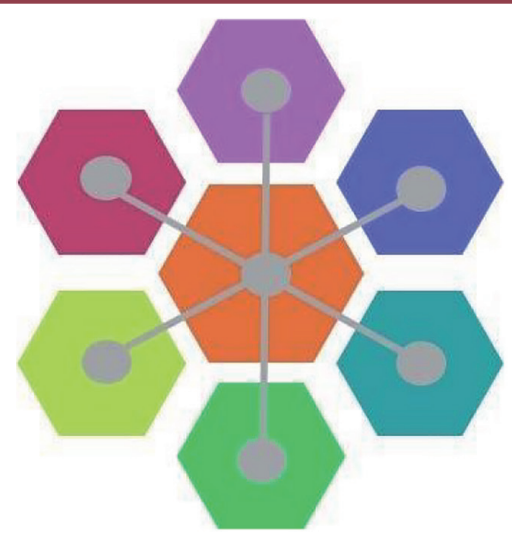

\section{Globall Registry for COL6-related dystrophies}

\section{Registre globall des dystrophies liées au collagène de type VI}

S'inscrire sur : www.collagen6.org

Ou contactez-nous par e-mail à l'adresse : collagenbregistry@ncl.ac.uk

La traduction française sera bientôt disponible sur le site web.
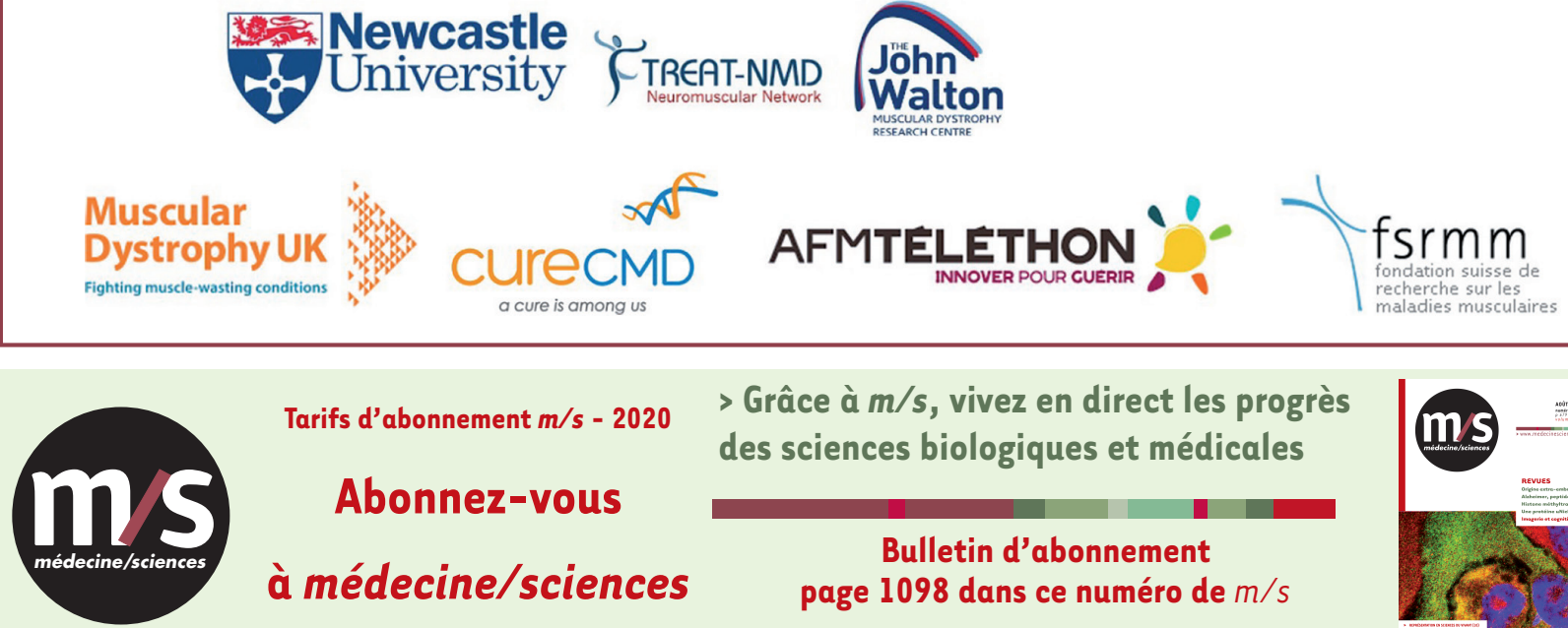

Tarifs d'abonnement $\mathrm{m} / \mathrm{s}-2020$

$>$ Grâce à $m / s$, vivez en direct les progrès des sciences biologiques et médicales

Abonnez-vous

à médecine/sciences

Bulletin d'abonnement page 1098 dans ce numéro de $\mathrm{m} / \mathrm{s}$
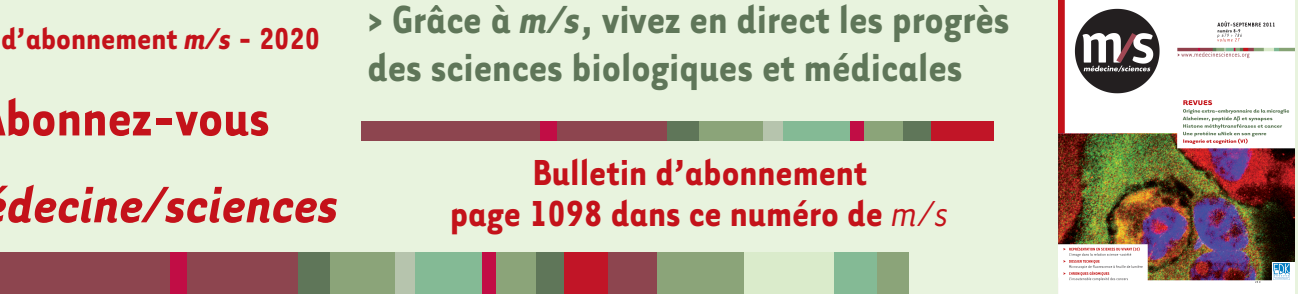